\title{
MOLECULAR DYNAMICS SIMULATIONS OF SEVERAL SELECTED COMPOUNDS FROM THE HERBAL DATABASE OF INDONESIA RESULTS OF MOLECULAR DOCKING AGAINST DNA METHYLTRANSFERASE ENZYME
}

\author{
MUHAMAD FIKRI IHSAN, ARRY YANUAR* \\ Department of Biomedical Computation, Faculty of Pharmacy, Universitas Indonesia, Depok, 16424, Indonesia. \\ Email: arry.yanuar@ui.ac.id
}

Received: 20 July 2018, Revised and Accepted: 29 September 2018

\begin{abstract}
Objective: This study aimed to investigate the interactions of DNA methyltransferase (DNMT) enzymes and potential ligands as DNMT inhibitors through molecular dynamics simulations.

Methods: This study was conducted using tools in the form of hardware (primary and secondary computers) and software (OpenBabel, AutoDock Tools, Amber MD, Amber Tools, VMD, PuTTY, LigandScout, and UCSF Chimera).

Results: Results of molecular docking of cassiamin C, procyanidin B2, epicatechin-4alphaent-8-ent-epicatechin, epicatechin-4beta-8-epicatechin3-O-gallate, neorhusflavanone, 3-O-galloylepigallocatechin -4beta-6-epicatechin-3-O-gallate, withanolide, 3-O-galloylepigallocatechin-4beta-6epigallocatechin-3-0-gallate, cyanidin-3-6"-caffeylsophoroside-5-glucoside, epifriedelinol, gallocatechin-4alpha-8-epicatechin, scutellarein-7glucosyl-1-4-rhamnoside, epigallocatechin-3-gallate (EGCG) (positive control), and sinefungin (co-crystal) compounds showed $\Delta \mathrm{G}$ values -9.34, $-10.95,-7.95,-11.01,-8.78,-8.87,-11.49,-7.98,-5.92,-8.92,-9.17,-8.76,-9.70$, and $-9.11 \mathrm{kcal} / \mathrm{mol}$, respectively. Cassiamin C, procyanidin B2, epicatechin-4-beta-8-epicatechin-3-O-gallate, withanolide, and gallocatechin-4alpha-8-epicatechin compounds had lower $\Delta G$ than sinefungin (cocrystal) and EGCG (positive control) compounds. The results of molecular dynamic simulation of seven selected compounds showed the best overall activities were procyanidin B2, epicatechin-4beta-8-epicatechin-3-O-gallate, and gallocatechin-4alpha-8-epi-catechin compounds.
\end{abstract}

Conclusions: The best overall activities based on molecular docking and molecular dynamic simulation were procyanidin B2, epicatechin-4beta8-epicatechin-3-O-gallate, and gallocatechin-4alpha-8-epi-catechin compounds. Amino acid residues that are important for the activity of DNMT1 inhibitor are Phe1145, Glu1168, Met1169, Cys1191, Glu1266, Ala1579, and Val1580.

Keywords: Cancer, DNA Methyltransferase, Epigenetic, Herbal database Indonesia, Molecular dynamic simulation.

(c) 2018 The Authors. Published by Innovare Academic Sciences Pvt Ltd. This is an open access article under the CC BY license (http://creativecommons. org/licenses/by/4. 0/) DOI: http://dx.doi.org/10.22159/ijap.2018.v10s1.63

\section{INTRODUCTION}

Cancer is a disease characterized by abnormal uncontrolled growth of body cells, especially with respect to cell growth and differentiation, and can affect other body tissues through the circulatory system and the lymphatic system $[1,2]$. Cancer is one of the leading causes of death worldwide. In 2012, about 8.2 million deaths were caused by cancer. It has also been estimated that there may be an increase in cancer patients in Indonesia, because of lifestyle patterns including increases in cigarette use, alcohol consumption, and lack of physical activity. One important factor in cancer occurrence is the abnormal epigenetic modification of DNA known as hypermethylation [3]. Hypermethylation of DNA can lead to the suppression of genes that should not be suppressed and is a risk factor for non-communicable diseases such as cancer [4-6]. No therapy currently exists that can restore abnormal gene expression, which is one of the main cancer risk factors [7]. Hypermethylation occurring in suppressor genes is believed to have a major role in the process of carcinogenesis through the increased expression of DNA methyltransferases (DNMTs) [8]. Therefore, one way to restore the function of suppressed genes is to use drugs that act to inhibit DNMTs $[8,9]$. DNMT inhibitors (DNMTis) can take the form of nucleoside and non-nucleoside analogs [9-11].

Compounds from natural substances are some of the most promising non-nucleoside DNMTi sources because they have high levels of diversity and tend to have low toxicity $[12,13]$. The exploration of natural material compounds for their potential use as DNMTis was previously carried out by Wilaputraka using the in silico method of molecular docking [6]. These results showed that there were 12 chemical compounds from Indonesia's herbal databases that could potentially serve as DNMTis based on their binding energies, with binding energies lower than a cocrystal ligand [6]. However, this analysis did not clearly examine binding stability in space and time. Therefore, it is necessary to simulate the molecular dynamics to further investigate the interactions and stability of the bindings. In this study, we investigated the interactions of DNMT enzymes and potential ligands as DNMTi through molecular dynamics simulations. The ligands used were derived from the results of a virtual screening of chemical compounds from Indonesian herbal databases conducted by Wilaputraka [6]. As a positive control, we used epigallocatechin-3-gallate (EGCG), which has been shown to function as a non-nucleoside DNMTi $[6,14-16]$.

\section{METHODS}

This study was conducted using tools in the form of hardware and software. The hardware used consisted of three primary computers (servers) and three secondary computers (clients) with different specifications. The first primary (server) computer with Intel ${ }^{\circledR}$ Xeon E5620 (Intel ${ }^{\circledR}$ Core $^{\mathrm{TM}}$, American) processor (CPU), Nvidia ${ }^{\circledR}$ GeForce GTX 780 (Nvidia ${ }^{\circledR}$, American) graphics processing unit (GPU), and random-access memory (RAM) of 32 gigabyte (GB) DDR3. The server ran the Ubuntu Linux operating system 12.04 LTS. The second and third servers had almost identical specs, Intel ${ }^{\circledR}$ Core $^{T M}$ i7-3770 (Intel $^{\circledR}$ Core $^{\mathrm{TM}}$, American) CPUs, 16GB of DDR3 RAM, and run 64-bit Debian 7.8 
Linux operating system. However, both servers used different GPUs. The second server used the Nvidia ${ }^{\circledR}$ GeForce GTX 970 (Nvidia ${ }^{\circledR}$, USA) GPU, while the third server used the Nvidia ${ }^{\circledR}$ GeForce GTX 980 (Nvidia ${ }^{\circledR}$, USA) GPU. All servers were connected with internet connection and uninterrupted power supply.

The first client computer used Intel ${ }^{\circledR} \operatorname{Core}^{\mathrm{TM}} 2$ Quad Q9400 (Intel ${ }^{\circledR} \mathrm{Core}^{\mathrm{TM}}$, USA) CPUs, Nvidia ${ }^{\circledR}$ GeForce GT 9400 (Nvidia ${ }^{\circledR}$, USA) GPUs, and 4 GB of DDR2 RAM. The computer ran the Linux operating system Ubuntu 12.04 LTS that is equipped with monitor (AOC, China), mouse (Logitech, China), and keyboard (HP, China). The second client computer was Mac Mini (Apple Inc., USA) with Intel ${ }^{\circledR}$ Core $^{\mathrm{TM}}$ i5-2450M (Intel ${ }^{\circledR}$ Core $^{\mathrm{TM}}$, USA) CPUS, Intel ${ }^{\circledR}$ Iris 1536 MB (Intel® ${ }^{\circledR}$ Core $^{\mathrm{TM}}$, USA) GPUs, and 8 GB of RAM DDR3. The computer ran the Yosemite $10.10 \mathrm{X}$ operating system equipped with monitors (AOC, China), as well as a mouse and keyboard (Apple Inc., USA). The third client computer was Asus ${ }^{\circledR}$ A46CM (Asus ${ }^{\circledR}$, Taiwan) with Intel ${ }^{\circledR}$ Core $^{\mathrm{TM}}$ i5-3317U (Intel ${ }^{\circledR}$ Core $^{\mathrm{TM}}$, American) CPU specifications, Nvidia ${ }^{\circledR}$ GeForce GT 635M (Nvidia ${ }^{\circledR}$, USA) GPUs, and 8 GB of RAM DDR3. The computer ran Microsoft Windows 10 Pro 64-bit (USA) operating system equipped with Logitech MK240 mouse and keyboard (Logitech, China). All clients were connected to an internet connection.

The software used for our analyses included OpenBabel (Hutchison et al.), AutoDock Tools (Scripps Research Institute, USA), Amber MD (University of California, San Francisco, USA), Amber Tools (University of California, San Francisco, USA), VMD (University of Illinois at UrbanaChampaign, USA), PuTTY (UK), LigandScout (InteLigand, Austria), and UCSF Chimera (University of California, San Francisco, USA). The materials used were a three-dimensional (3D) target macromolecular structure and a 3D ligand structure. The 3D structure of DNMT1 was downloaded from the Research Collaboratory for Structural Bioinformatics Protein Data Bank (RCSB PDB, at http://www.rcsb. org/pdb), with the ID number 3SWR. This structure had a resolution of $2.49 \AA$ with a sinefungin cocrystal ligand. The 3D ligand structure used was the result of a previous virtual screening study conducted by Wilaputraka [6]. The ligands (Table 1) were obtained and downloaded from the HerbalDB database, KNApSAcK, ChemSpider, and PubChem.

The first step in our analysis was to search for 3D ligand and target macromolecule structures that could be downloaded from the RCSB GDP database, HerbalDB, KNApSAcK, PubChem, and ChemSpider. Then, preparation and optimization of target ligands and macromolecules using OpenBabel, AutoDock Tools, Antechamber, and Sander were performed. After that, molecularly docked ligands were made against the target macromolecules using the AutoDock $\mathrm{Zn}$ method and designed specifically for target macromolecules that contain and have interactions with zinc atoms. Molecular docking was performed using a $60 \times 60 \times 60 \AA$ gridbox, centered on the coordinates $x=-5.041, y=-0.949$, and $z=31.815$, with spacing of $0.375 \AA$. The molecular docking of the ligand compound had a lower binding energy than the crystal ligand

\section{Table 1: Ligand compounds}

\begin{tabular}{ll}
\hline Code & Ligand \\
\hline A & Cassiamin C \\
B & Procyanidin B2 \\
C & Ent-epicatechin-4alpha-8-ent-epicatechin \\
D & Epicatechin-4beta-8-epicatechin-3-0-gallate \\
E & Neorhusflavanone \\
F & 3-O-galloylepicatechin-4beta-6-epicatechin-3-0-gallate \\
G & Withanolide \\
H & 3-O-galloylepigallocatechin-4beta-6-epigallocatechin-3-0-gallate \\
I & Cyanidin-3-6"-caffeylsophoroside-5-glucoside \\
J & Epifriedelinol \\
K & Gallocatechin-4alpha-8-epicatechin \\
L & Scutellarein-7-glucosyl-1-4-rhamnoside \\
EGCG & Epigallocatechin-3-gallate (EGCG) (Positive Control) \\
SFG & Sinefungin (Co-crystal ligand) \\
\hline
\end{tabular}

and the positive control ligand, and we simulated molecular dynamics for 20 ns to examine the stability of the compound conformation and binding in space and time. Simulation of molecular dynamics was done using AMBER 12. The results of molecular dynamics simulations were analyzed in terms of root mean square deviation (RMSD), root mean square fluctuation (RMSF), hydrogen binding, and binding energy of the amino acid residues of macromolecules and ligands by MMPBSA/ MMGBSA method.

\section{RESULTS}

\section{Molecular docking}

The results from molecular docking of the ligand to DNMT1 macromolecule using the AutoDock $\mathrm{Zn}$ method (Table 2) found five compounds whose binding affinity and inhibitor constant Ki were lower than those of the crystalline ligand (sinefungin) and the positive control ligand (EGCG). We refer to these as ligand A (cassiamin C), ligand B (procyanidin B2), ligand D (epicatechin-4beta-8-epicatechin-3-Ogallate), ligand G (withanolide), and ligand $\mathrm{K}$ (gallocatechin-4alpha8-epicatechin). Thus, we selected these ligands to perform molecular dynamics simulations for their potential to be DNMTis. Negative bonding energy of a compound to the target macromolecule indicates that the energy required by the ligand to bind to the macromolecule is lower, while a low value of the inhibition constant Ki indicates inhibitory activity of the ligand toward the target protein.

\section{Molecular dynamics simulations \\ RMSD}

In the molecular dynamics simulation of ligand compound with DNMT1 for $20 \mathrm{~ns}$, the RMSD fluctuation showed that the sinefungin cocrystal (SFG) ligand was stable at $1.5 \AA$ within 2 ns (Fig. 1). At $13 \mathrm{ns,}$ the RMSD value increased to $2 \AA$; however, after $14.5 \mathrm{~ns}$, the system returned to stability at RMSD $1.5 \AA$. The same happened with the ligand B (procyanidin B2), which started stable at $2 \AA$ RMSD within $4.5 \mathrm{~ns}$, increased in RMSD to $2.5 \AA$ at $13 \mathrm{~ns}$, and then returned to stability at $2 \AA$ RMSD after $14.5 \mathrm{~ns}$. The ligand D (epicatechin-4beta-8-epi-catechin-3$O$-gallate) showed similar behavior, in which the RMSD values were stable at below $2 \AA$ a within 3 ns. However, at 12 ns, the system continued to increase in RMSD value up to $3 \AA$. After the system passed $19 \mathrm{~ns}$, the system returned to stable on RMSD $2 \AA$ up to $20 \mathrm{~ns}$. The positive control EGCG ligand showed a stable RMSD value at $2 \AA$ within $13.5 \mathrm{~ns}$ and continued to stabilize up to $20 \mathrm{~ns}$. A similar thing happened with ligand A (cassiamin C), which started stable at RMSD $1.5 \AA$ A within $17 \mathrm{~ns}$ and kept stable up to $20 \mathrm{~ns}$. Ligand G (withanolide) also behaved similarly in which the RMSD value began to stabilize at below $2 \AA$ within $10 \mathrm{~ns}$ and continue to stabilize up to $20 \mathrm{~ns}$. The ligand $\mathrm{K}$ (gallocatechin-4alpha8-epicatechin) behaved differently, where the RMSD values began to stabilize at $2 \AA$ within $6.5 \mathrm{~ns}$. At $15 \mathrm{~ns}$, the RMSD value continued to increase up to $2.5 \AA$, and the system returned to stable at RMSD $2 \AA$ after 16 ns. However, at $18.5 \mathrm{~ns}$, the RMSD value increased above $2.5 \AA$ and the system did not return to stability until the 20 -ns simulation time was complete.

An increase in RMSD value indicates the effect of time on binding stability. Thus, it was necessary to lengthen the simulation time to see if the molecular dynamics further changed in terms of the stability of conformational compounds. In addition, one limitation of this study was that simulations were only performed at room temperature settings of $27^{\circ} \mathrm{C}$ (or $300 \mathrm{~K}$ ). Thus, we could not examine changes in the stability of conforming compounds at different temperatures. Therefore, in future work, it will be necessary to simulate molecular dynamics at different temperatures, such as the human body temperature of $\pm 37^{\circ} \mathrm{C}$, where drug-receptor reactions and interactions occur, or higher temperatures.

\section{RMSF}

High values of RMSF occurred at amino acid residues $640-700,840-$ 880, 940-1020, 1080-1120, 1460-1500, and 1590-1600 (Fig. 2). Amino acid residues with high RMSF values are highly flexible and 


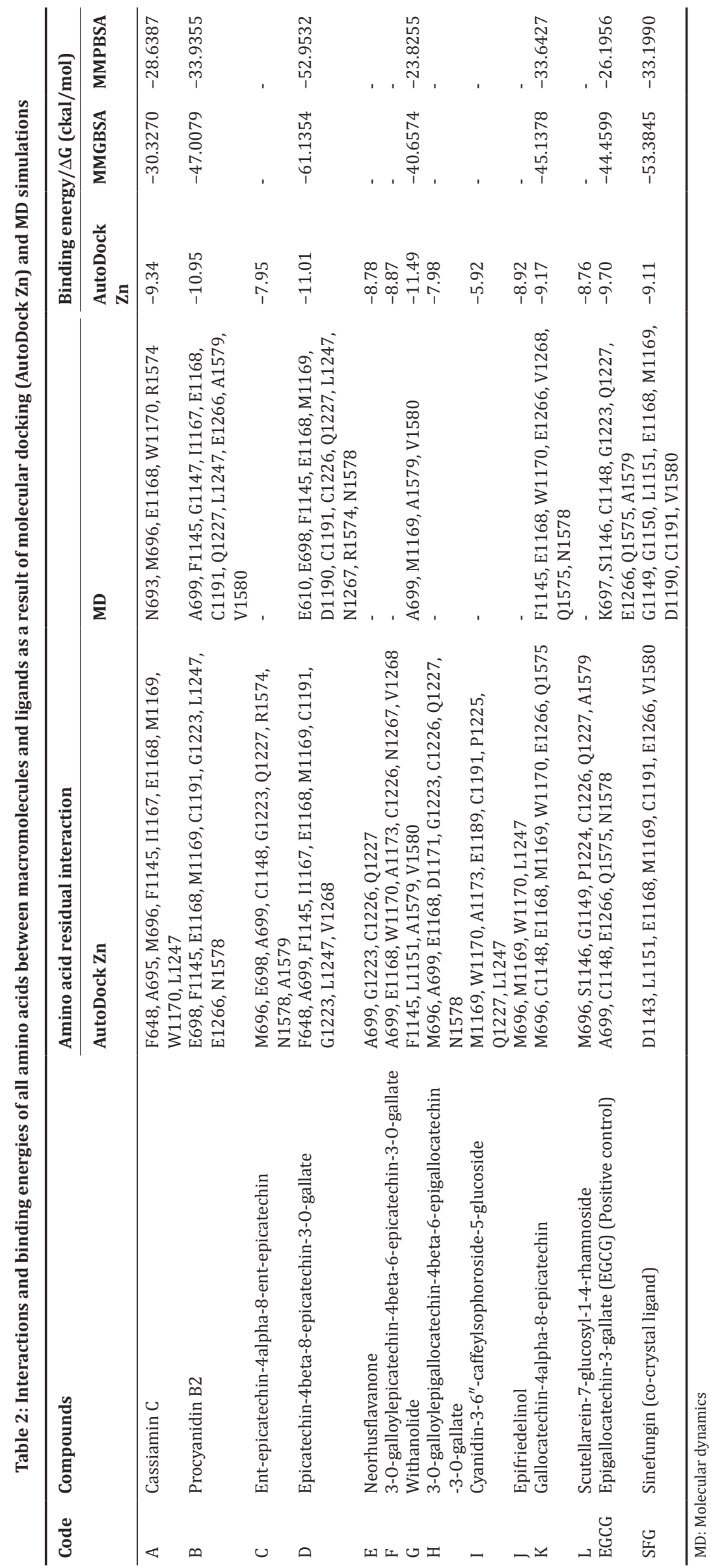




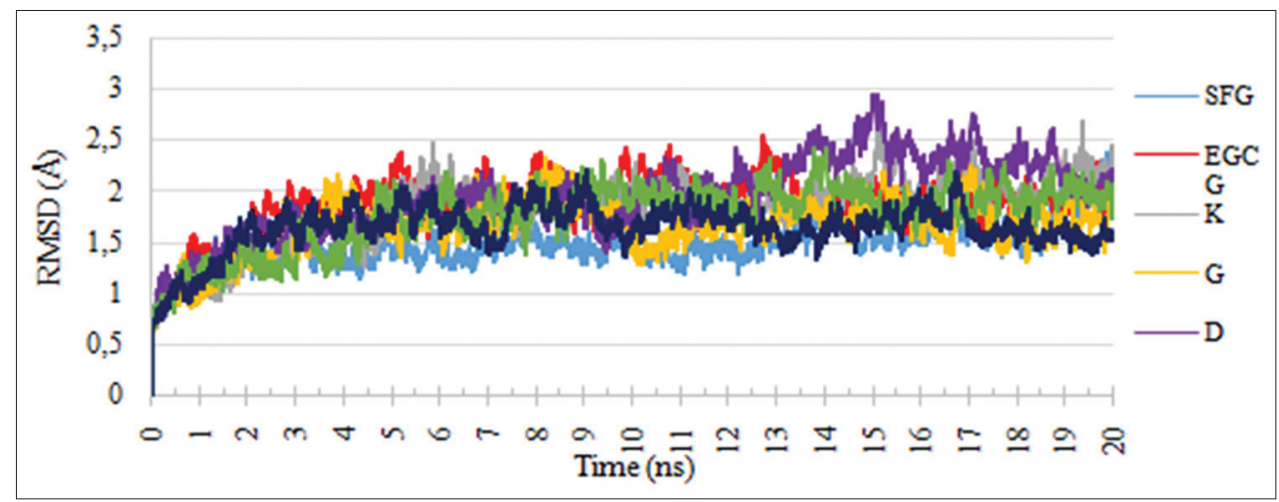

Fig. 1: Graph of root mean square deviation fluctuations in molecular dynamics simulations of ligand compounds with DNA methyltransferase 1 for 20 ns

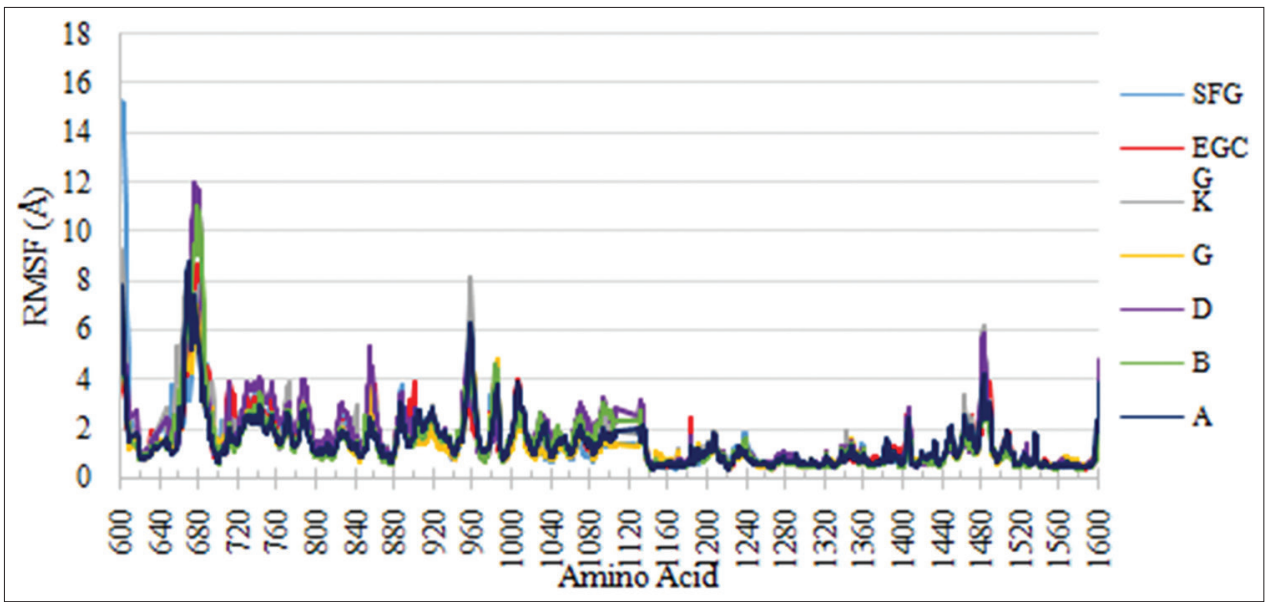

Fig. 2: Graph of root mean square deviation fluctuations in molecular dynamics simulations of ligand compounds with DNA methyltransferase 1 for $20 \mathrm{~ns}$

tend to be unstable. In addition, in these residues, the ligand was most likely to change position. The active sites of ligands on the amino acid residues Phe1145, Ser1146, Gly1147, Gly1149, Gly1150, Leu1151, Glu1168, Met1169, Trp1170, Asp1190, Cys1191, Pro1225, Leu1247, Glu1266, Asn1578, Ala1579, and Val1580 showed lower RMSF values. This indicates that these amino acid residues provided lower flexibility, and the interaction between the ligand and the residue tended to be stable.

\section{Hydrogen bonds}

The SFG cocrystal ligand (sinefungin) and ligand D (epicatechin-4beta-8epicatechin-3-0-gallate) had hydrogen bonds in the range of 20-30. The ligand B (procyanidin B2) had a hydrogen bond in the range of 15-30. The positive control EGCG ligand and ligand K (gallocatechin-4alpha-8epi-catechin) had hydrogen bonds in the range of 10-20. The ligand A (cassiamin C) had a hydrogen bond in the range of 5-10, while the ligand G (withanolide) had the fewest hydrogen bonds, in the range of 0-5 (Fig. 3).

In terms of the percentage of hydrogen bond occupancy that occurred during the molecular dynamics simulation, all of the ligands occupied the side of the corresponding active sites and showed stable hydrogen bonds on the active residues Phe1145, Ser1146. Gly1147, Glu1168, Asp1190, Cys1191, Glu1266, and Asn1578 (Table 3). However, for the ligand G (withanolide) and ligand $\mathrm{K}$ (gallocatechin-4alpha-8epicatechin), the hydrogen bonds did not show a stable occupancy value in active residues but were stable in the non-active amino acid residues Met696, Gly1223, Cys1226, Asn1267, and Gln1575. The same amino acid residual involvement showed the extent of the ligand binding tendency to the macromolecule. The occupancy result of ligand A (cassiamin C), ligand B (procyanidin B2), and ligand D (epicatechin-4beta-8-epicatechin-3-0-gallate) showed similarities with EGCG (positive control) compounds and sinefungin (co-crystal ligand) because they had a common binding site. This suggests that these ligand compounds most likely have similar activity to EGCG and sinefungin as compound inhibitors of the enzyme DNMT1.

\section{MMPBSA/MMGBSA}

The result of calculation of binding energy by MMPBSA/MMGBSA method (Table 2) showed that the value of binding energy resulted from dynamic simulation with MMPBSA/MMGBSA was always lower than binding energy of molecular docking value, since the calculation method of $\Delta \mathrm{G}$ with molecular dynamics had smaller calculation error value (accurate). In addition, from MMGBSA results obtained three ligands that had lower binding energy than EGCG-positive control ligand, the ligands were ligand B (procyanidin B2), ligand D (epicatechin4beta-8-epicatechin-3-O-gallate), and ligand $\mathrm{K}$ (gallocatechin-4alpha8-epicatechin). From MMPBSA results obtained, four ligands that had lower binding energy than EGCG-positive control ligand, were ligand A (cassiamin C), ligand B (procyanidin B2), ligand D (epicatechin4beta-8-epicatechin-3-O-gallate), and ligand $\mathrm{K}$ (gallocatechin-4alpha8-epicatechin). It demonstrated the potency of these ligands to be able to have activity similar as EGCG-positive control ligand that had proven in silico and in vitro had activity as a non-nucleoside DNMTi [6,14-16].

The result of calculation of MMPBSA/MMGBSA binding energy on ligand $\mathrm{G}$ (withanolide) did not show lower binding energy value than EGCG-positive control ligand. However, it did not rule out that the compound of withanolide ligand could have thr activity as DNMTi similar with EGCG-positive control ligand, since the binding energy 


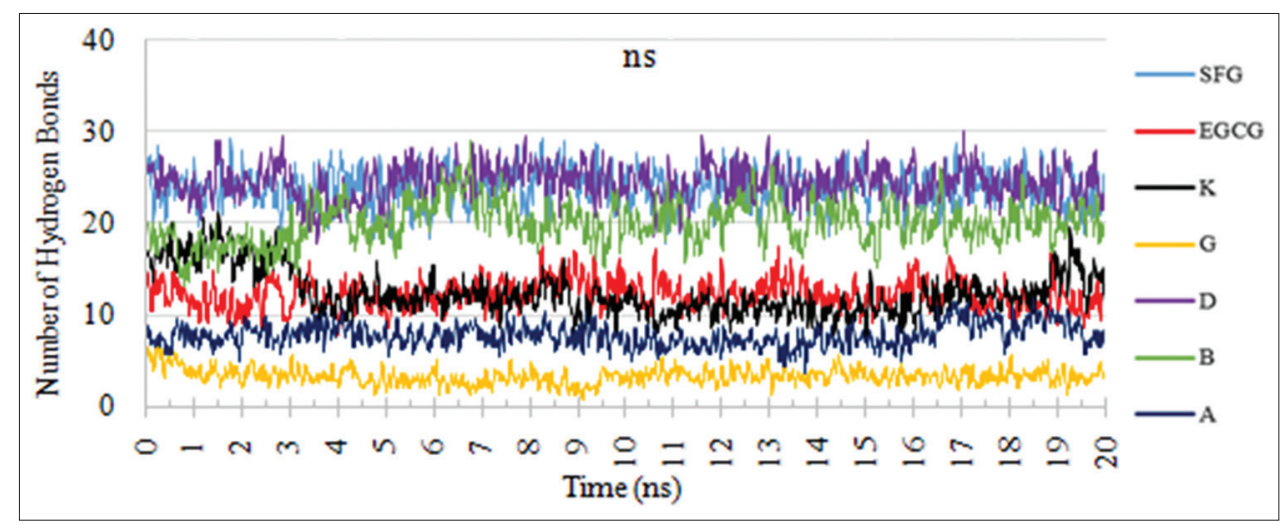

Fig. 3: Graph of fluctuations in the number of hydrogen bonds in molecular dynamics simulations of ligand compounds with DNA methyltransferase 1 for $20 \mathrm{~ns}$ in five moving average periods

Table 3: Percentage occupancy of hydrogen binding of macromolecular ligands complex

\begin{tabular}{|c|c|c|c|c|c|}
\hline Code & Compounds & Donor & Acceptor & Binding distance $(\AA ̊)$ & Occupancy (\%) \\
\hline \multirow[t]{4}{*}{ A } & \multirow[t]{4}{*}{ Cassiamin C } & Arg1574-Side-NH1 & Lig-Side-03 & 2.169 & 52.35 \\
\hline & & Lig-Side-06 & Glu1168-Side-OE1 & 1.952 & 91.95 \\
\hline & & Lig-Side-07 & Glu1168-Side-OE1 & 1.954 & 92.20 \\
\hline & & Phe1145-Main-N & Lig-Side-07 & 2.698 & 91.65 \\
\hline \multirow[t]{9}{*}{ B } & \multirow[t]{9}{*}{ Procyanidin B2 } & Cys1191-Main-N & Lig-Side-06 & 2.236 & 89.75 \\
\hline & & Lig-Side-011 & Gln1227-Side-OE1 & 1.783 & 51.85 \\
\hline & & Lig-Side-01 & Phe1145-Main-0 & 1.848 & 86.55 \\
\hline & & Lig-Side-09 & Glu1168-Side-OE2 & 2.128 & 58.90 \\
\hline & & Lig-Side-08 & Gly1147-Main-0 & 1.713 & 78.80 \\
\hline & & Lig-Side-O2 & Glu1266-Side-0E1 & 1.723 & 98.85 \\
\hline & & Lig-Side-03 & Glu1266-Side-OE1 & 1.637 & 100.00 \\
\hline & & Arg1312-Side-NH1 & Lig-Side-03 & 3.194 & 63.05 \\
\hline & & Cys1191-Side-SG & Lig-Side-06 & 2.375 & 54.80 \\
\hline \multirow[t]{10}{*}{$\mathrm{D}$} & \multirow[t]{10}{*}{ Epicatechin-4beta-8-epicatechin-3-0-gallate } & Cys1226-Main-N & Lig-Side-012 & 2.478 & 91.35 \\
\hline & & Cys1191-Main-N & Lig-Side-03 & 2.314 & 85.85 \\
\hline & & Lig-Side-07 & Glu698-Side-OE2 & 1.683 & 89.40 \\
\hline & & Lig-Side-012 & Asn1267-Side-OD1 & 2.194 & 95.85 \\
\hline & & Lig-Side-02 & Glu1168-Side-OE2 & 1.777 & 99.90 \\
\hline & & Lig-Side-05 & Glu1168-Side-0E1 & 1.734 & 100.00 \\
\hline & & Lig-Side-01 & Gly1223-Main-0 & 1.800 & 99.90 \\
\hline & & Lig-Side-04 & Asp1190-Side-OD2 & 1.847 & 100.00 \\
\hline & & Lig-Side-03 & Asp1190-Side-OD2 & 1.716 & 99.95 \\
\hline & & Lig-Side-04 & Asp1190-Side-CG & 3.251 & 73.65 \\
\hline G & Withanolide & Gly1223-Main-N & Lig-Side-01 & 1.921 & 66.35 \\
\hline \multirow[t]{4}{*}{$\mathrm{K}$} & \multirow[t]{4}{*}{ Gallocatechin-4alpha-8-epicatechin } & Lig-Side-01 & Gln1575-Main-0 & 1.667 & 97.65 \\
\hline & & Cys1226-Main-N & Lig-Side-08 & 4.357 & 81.10 \\
\hline & & Lig-Side-08 & Asn1267-Side-OD1 & 2.424 & 82.90 \\
\hline & & Met696-Main-N & Lig-Side-09 & 3.348 & 50.25 \\
\hline \multirow[t]{4}{*}{ EGCG } & \multirow[t]{4}{*}{ Epigallo-catechin-3-gallate (positive control) } & Asn1267-Side-CA & Lig-Side-O3 & 3.079 & 78.20 \\
\hline & & Lig-Side-02 & Gly1223-Main-0 & 1.699 & 98.05 \\
\hline & & Lig-Side-07 & Gln1575-Main-0 & 1.904 & 91.35 \\
\hline & & Lig-Side-03 & Glu1266-Side-0E1 & 1.748 & 100.00 \\
\hline \multirow{3}{*}{ SFG } & \multirow{3}{*}{ (co-crystal ligand) } & Lig-Side-CD & Phe1145-Main-0 & 4.615 & 53.00 \\
\hline & & Lig-Side-N6 & Asp1190-Side-OD2 & 1.623 & 97.50 \\
\hline & & Lig-Main-CA & Ser1146-Main-0 & 3.818 & 72.70 \\
\hline
\end{tabular}

value in the ligand did not differ greatly with the binding energy value in the EGCG-positive control ligand.

\section{DISCUSSION}

From the results of molecular dynamics simulations on the DNMT1 macromolecule (Table 2), we identified three compounds with the best activity: Procyanidin B2, epicatechin-4beta-8-epicatechin-3-O-gallate, and gallocatechin-4-alpha-8-epicatechin. These three compounds showed a stable bond on the active residue (binding site) based on the RMSD and RMSF values. In addition, these three compounds had a number of hydrogen bonds similar to or greater than the EGCG-positive control ligand, had a high occupancy hydrogen bond, and belonged to a very strong category of hydrogen bonding (above 50\%). Where the formation of hydrogen bonds with amino acids on the active (binding) 
site was the mechanism of the action of non-nucleoside DNMTi $[6,14]$. Then, from the calculation of binding energy by MMPBSA/MMGBSA, the three compounds also had lower binding energy than EGCG-positive control ligand, which means that these three compounds had the similar or even better potency from EGCG that had proven in silico and in vitro had the activity as a non-nucleoside DNMTi [6,14-16]. In addition, from the visualization of ligand interaction with metromagnetic DNA macromolecule 1 (DNMT1), the three compounds also showed the interaction of active residue (binding) corresponding to the active residue (binding) in the sequence (RCSB GDP). Further research is needed, both in vitro and in vivo, to test the inhibitory activity of ligand compounds on DNMT enzymes.

\section{CONCLUSIONS}

Cassiamin C compounds, procyanidin B2, epicatechin-4beta-8epicatechin-3-O-gallate, withanolide, and gallocatechin-4alpha-8epicatechin had lower binding energy values $/ \Delta G$ than sinefungin (co-crystal) and EGCG (positive controls). Thus, molecular dynamics simulations showed the best overall activity of procyanidin B2, epicatechin-4beta-8-epicatechin-3-O-gallate, and gallocatechin-4alpha8-epicatechin. Amino acid residues that were important for the activity of DNMT1 inhibitors were Phe1145, Glu1168, Met1169, Cys1191, Glu1266, Ala1579, and Val1580.

\section{ACKNOWLEDGMENT}

All authors acknowledge Universitas Indonesia for support and PUPT Research Grants 2018.

\section{CONFLICTS OF INTEREST}

The authors have no conflicts of interest.

\section{REFERENCES}

1. Maliya A. Cell changes Become Cancerous from the Point of View of Molecular Biology. Vol. 1. Indonesia: Infokes; 2004. p. 1-7.

2. American Cancer Society. The History of Cancer. American Cancer Society; 2014. p. 1-16. Available from: http://www.cancer.org/thehistory-of-cancer-pdf. [Last accessed on 15 Sep 2016].

3. Luczak MW, Jagodziński PP. The role of DNA methylation in cancer development. Folia Histochem Cytobiol 2006;44:143-54.
4. Mikeska T, Craig JM. DNA methylation biomarkers: Cancer and beyond. Genes (Basel) 2014;5:821-64.

5. Lao TD, Le TA. Hypermethylated DNA as biomarker for nasopharyngeal cancer detection. Asian J Pharm Clin Res 2018;11:68-71.

6. Wilakaputra IGNRB. Virtual Screenig of Indonesian Herbal Database Inhibitor DNA Metiltransferase. Depok: Faculty of Pharmacy, Universitas Indonesia; 2016.

7. Yoo CB, Jones PA. Epigenetic therapy of cancer: Past, present and future. Nat Rev Drug Discov 2006;5:37-50.

8. Gnyszka A, Jastrzebski Z, Flis S. DNA methyltransferase inhibitors and their emerging role in epigenetic therapy of cancer. Anticancer Res 2013;33:2989-96.

9. Rius M, Lyko F. Epigenetic cancer therapy: Rationales, targets and drugs. Oncogene 2012;31:4257-65.

10. Lyko F, Brown R. DNA methyltransferase inhibitors and the development of epigenetic cancer therapies. J Natl Cancer Inst 2005;97:1498-506.

11. Yen CY, Huang HW, Shu CW, Hou MF, Yuan SS, Wang HR, et al. DNA methylation, histone acetylation and methylation of epigenetic modifications as a therapeutic approach for cancers. Cancer Lett 2016;373:185-92.

12. Medina-Franco JL. Advances in computational approaches for drug discovery based on natural products. Rev Latinoam Quim 2013;41:95-110

13. Maldonado-Rojas W, Olivero-Verbel J, Marrero-Ponce Y. Computational fishing of new DNA methyltransferase inhibitors from natural products. J Mol Graph Model 2015;60:43-54.

14. Singh BN, Shankar S, Srivastava RK. Green tea catechin, epigallocatechin-3-gallate (EGCG): Mechanisms, perspectives and clinical applications. Biochem Pharmacol 2011;82:1807-21.

15. Gros C, Fahy J, Halby L, Dufau I, Erdmann A, Gregoire JM, et al. DNA methylation inhibitors in cancer: Recent and future approaches. Biochimie 2012;94:2280-96.

16. Shilpi A, Parbin S, Sengupta D, Kar S, Deb M, Rath SK, et al. Mechanisms of DNA methyltransferase-inhibitor interactions: Procyanidin B2 shows new promise for therapeutic intervention of cancer. Chem Biol Interact 2015;233:122-38. 